\title{
Práticas de lazer, nível de atividade física e aptidão física de moças e rapazes Brasileiros
}

\author{
Brazilian girls' and boys' leisure practices, physical activity level \\ and physical fitness
}

\author{
Francisco Salviano Sales-Nobre ${ }^{1}$, Ruy Jornada-Krebs ${ }^{2}$ e Nadia C. Valentini ${ }^{3}$ \\ 1 Departamento de Educação Física. Instituto Federal de Educação, Ciência e Tecnologia do
Ceará-IFETCE. Brasil. salvianonobre @ cefetce.br
2 Centro de Educação Física, Fisioterapia e Desporto-CEFID. Universidade do Estado de Santa
Catarina-UDESC. Brasil. ruykrebs@ brturbo.com.br
3 Escola Superior de Educação Física. Universidade Federal do Rio Grande do Sul-UFRGS. Brasil.
nadiacv@esef.ufrgs.br
}

Recebido em 15 Dezembro 2008/Enviado para Modificação em 6 Julho 2009/Aprovado 29 Agosto 2009

\section{RESUMO}

Objetivo Identificar os hábitos de lazer, o nível de atividade física e a aptidão física relacionada à saúde de adolescentes brasileiros de ambos os sexos.

Material e Método Participaram do estudo 222 rapazes e 152 moças, com idade entre 15 e 18 anos. Os instrumentos de coleta foram: Inventaire d'habitudes du loisir (adaptado para língua portuguesa) e a Escala de Hábitos de Lazer. O nível de atividade física foi avaliado por meio do Iternational Physical Actitivity Questionnarie, versão curta, e o nível de aptidão física foi avaliado através da bateria de testes e medidas proposta pelo Projeto Esporte Brasil. A análise dos dados foi feita através da distribuição de freqüência, média e desvio padrão e por meio do Teste $U$ de Mann Whitney e ANOVA.

Resultados Os resultados mostraram que não existe relação linear entre hábitos de lazer sedentário e aptidão física relacionada à saúde. Tanto os rapazes quanto as moças se consideraram fisicamente ativos, verificando-se, porém, uma maior predisposição dos rapazes para a prática do lazer ativo nos momentos de tempo livre, com uma conseqüente melhor aptidão física relacionada à saúde.

Conclusões Concluise que mesmo os adolescentes de hábitos de lazer fisicamente ativo não necessariamente alcançarão resultados satisfatórios nas qualidades físicas que exijam movimento ativo (resistência aeróbica e força/resistência abdominal) e, também, que a implementação de programas de lazer e de condicionamento físico requer atividades específicas. Independentemente do gênero, os adolescentes necessitam de ambas as atividades.

Palavras Chave: Atividades de lazer, atividade motora, aptidão física (fonte: DeCS, BIREME).

\section{ABSTRACT}

Objectives Identifying Brazilian adolescents' leisure habits, physical activity level and physical fitness related to health. 
Material and Methods 222 male and 152 female adolescents with ages ranging from 15 to 18 participated in this study. The instruments used were the Inventaire d'habitudes $d u$ loisir (adapted to Portuguese) and a Scale of Leisure Habits. Physical activity level was measured by the International Physical Activity Questionnaire (short version) and physical fitness was assessed through a battery of questions proposed by the Brazilian Sports' Project. The Mann-Whitney U and ANOVA tests were used for frequency-data analysis and inferential statistics. Statistical significance was set at the $<0.05$ level.

Results The results revealed no linear relationship between adolescents' sedentary leisure habits and aptitude level related to health, thereby inferring that both male and female adolescents considered themselves to be physically active. However, a higher predisposition towards active leisure habits was found in male adolescents during their free time leading them to enjoy a better aptitude level related to health.

Conclusions It was concluded that even adolescents having physically active leisure habits will not necessarily achieve satisfactory results in physical abilities depending on active movement (aerobic fitness and abdominal strength and fitness) and, also, implementing leisure and fitness programmes requires specific activities. Independently of gender, adolescents need physical activity.

Key Words: Health care, population characteristics, physical fitness (source: MeSH, NLM).

\section{RESUMEN}

Actividad Fisica y Praticas de Recreación en Jueves de Brasil

Objetivos Identificar hábitos de ocio, niveles de actividad física y aptitud física en adolescentes brasileños.

Material y Métodos Un total de 222 adolescentes de sexo masculino y 152 de sexo femenino, de 15 a 18 años de edad, participaron en el estudio. Como instrumentos se utilizaron el Inventaire d'habitudes du loisir (adaptado al idioma portugués) y la Escala de Hábitos de Ocio. El nivel de actividad física se evaluó con la International Physical Actitivity Questionnarie-IPAQ, versión corta; el nivel de aptitud física se evaluó por medio de la batería de pruebas y medidas propuesta por el Proyecto de los Deportes Brasileño. Los datos se analizaron por medio de la prueba $U$ de Mann-Whitney y ANOVA.

Resultados No se encontró ninguna relación linear entre los hábitos sedentarios y de ocio de adolescentes y el nivel de la aptitud física relacionada con la salud. Tanto los jóvenes como las jóvenes se consideraron físicamente activos, pero se encontró una mayor predisposición de los jóvenes para el ocio activo en tiempo libre, con una aptitud física más relacionada con la salud.

Conclusiones Los adolescentes con hábitos de ocio físicamente activos no necesariamente lograrán resultados satisfactorios en habilidades físicas que dependen en el movimiento activo (resistencia aeróbica y fuerza abdominal) y también que la implementación de programas de ocio y aptitud física requieren actividades específicas. Independiente del género, los adolescentes necesitaron ambas actividades.

Palabras Clave: Actividades recreativas, actividad motora, acondicionamiento físico (fuente: DeCS, BIREME). 
$\mathrm{D}$ urante esses últimos quinze anos a comunidade científica de todo o mundo tem alertado exaustivamente a população sobre a redução dos níveis de atividade física que vem ocorrendo na sociedade. Esse fenômeno ocorre independentemente do gênero ou raça, e tem sido associado às mudanças culturais advindas do progresso tecnológico (1). Estudos mais recentes desenvolvidos por meio do Inquérito Domiciliar sobre Comportamentos de Risco e Morbidade Referida de Doenças e Agravos não Transmissíveis (2), realizado em quinze capitais brasileiras e no Distrito Federal, apontam para uma distribuição média de inatividade física entre $27,4 \%$ e $55 \%$. Em Florianópolis-SC, capital onde se desenvolveu este estudo, constatou-se a terceira pior média de inatividade física $44,4 \%$.

A respeito da prática de atividades físicas, a grande maioria dos estudos revisados aponta para uma supremacia da participação do sexo masculino $(2,3$, $4,5,6)$. Essas diferenças registradas entre os sexos na predisposição da prática desportiva estão fortemente arraigadas a questões sócio-culturais. No entanto, tudo leva a crer que o fenômeno da menor participação feminina em atividades físicas deva ser melhor explicado, uma vez que estudos $(7,8)$ mostram uma igualdade e em alguns casos até superioridade do sexo feminino na prática da atividade física, principalmente quando estas estão relacionadas ao contexto laborativo e não ao cotidiano da prática esportiva no tempo livre. Diante da falta de unanimidade entre os autores no que diz respeito ao predomínio do sexo masculino em atividades físicas, bem como a necessidade de melhor explicar como esta se manifesta no cotidiano do adolescente, o presente trabalho teve como objetivo identificar os principais hábitos de lazer de adolescentes do sexo masculino e feminino em Florianópolis-SC, Brasil, e sua possível associação com o nível de atividade física e aptidão física relacionada à saúde.

\section{MATERIAL E MÉTODOS}

O estudo se caracterizou como sendo uma pesquisa descritiva, do tipo causal comparativa, considerando as variáveis: práticas de lazer, nível de atividade física e aptidão física relacionada à saúde norteadoras do estudo. $\mathrm{O}$ contexto aonde se desenvolveu a pesquisa foi o Centro Federal de Educação Tecnológica de Santa Catarina (CEFETSC), Unidade Florianópolis. Esta instituição possui jovens de diferentes extratos econômicos, sendo que ocorre uma prevalência de adolescentes de classes sócio-econômicas de nível médio e médio-baixo. Essa informação parece ser bastante importante uma vez que o nível socioeconômico exerce influências sobre as práticas de lazer e níveis de atividade física da população (9). 
A população do estudo compreendeu 537 adolescentes de ambos os sexos com faixa etária entre 15 e 18 anos, com média de idade de 16 anos e 10 meses, sendo 17 anos e 1 mês para os rapazes e 16 anos e 9 meses para as moças. A amostra foi formada por 374 adolescentes, sendo 222 rapazes e 152 moças. A opção pela faixa etária citada anteriormente foi intencional, devido à mesma ser referida na literatura como período crítico de mudanças de comportamento com consequiente abandono das atividades físicas $(5,10)$.

Este estudo foi desenvolvido de acordo com as Normas de Realização de Pesquisa em Seres Humanos, Resolução 196/96, do Conselho Nacional de Saúde, e avaliado pelo Comitê de Ética e Pesquisa da Universidade do Estado de Santa Catarina, pelo protocolo CEP no 96/2006 que aprovou a realização deste estudo. A coleta dos dados se deu nos meses de junho e julho de 2006.

A identificação dos hábitos de lazer foi realizada por meio do Inventaire d'habitudes du loisir (11) adaptado para língua portuguesa (12) e, da Escala de Hábitos de Lazer (13), os quais nortearam a elaboração do instrumento utilizado nesta pesquisa por Nobre. Esse instrumento é composto por 22 itens respondido em escala de cinco pontos, tipo Likert. Dessa forma, o quesito 1 diz respeito às atividades que definitivamente não fazem parte do dia a dia do adolescente; 2 raramente fazem parte do cotidiano; 3 as vezes; 4 quase sempre e 5 sempre. As alternativas de respostas dizem respeito a hábitos de lazer hedonistas, lúdicos e instrutivos que de certa forma representam as atividades preferidas por adolescentes nos seus momentos de tempo livre. As questões 3 e 21 do referido questionário dizem respeito a prática de atividades físicas/desportivas com e sem orientação profissional respectivamente, permitindo assim caracterizar os adolescentes como hábitos de lazer ativos ou sedentários.

Para avaliar o nível de atividade física dos adolescentes optou-se por se fazer uso do Iternational Physical Actitivity Questionnarie versão curta (IPAQ) validado para adolescentes brasileiros (14). A aptidão física foi avaliada por meio do protocolo desenvolvido pelo Projeto Esporte Brasil (PROESP-BR) (15). Nesses termos, para efeito desse estudo, as qualidades físicas avaliadas foram às relacionadas à saúde: teste de corrida e caminhada durante 9 minutos, o qual tem por objetivo avaliar a capacidade aeróbica, teste de sentar e alcançar (sit and reach), que se propõe avaliar a flexibilidade, o teste de flexão de tronco (sit-up) em 1 minuto que tem por objetivo avaliar a força-resistência de abdominais, e a avaliação da composição corporal por meio das medidas de peso, estatura, dobras cutâneas do tríceps e subescapular. Esse procedimento permitiu identificar o 
Índice de Massa Corporal-IMC e, o percentual de gordura dos adolescentes por meio do protocolo de Lohman (16).

O estudo se valeu da técnica estatística descritiva do tipo distribuição de freqüência, média e desvio padrão para analisar o perfil da amostra quanto suas características de sexo, idade, hábitos de lazer, nível de atividade física e, aptidão física relacionada à saúde. As diferenças inter e entre os grupos foi analisada por meio do Test U de Mann-Whitney para as variáveis qualitativas e análise de variância (ANOVA) para dados quantitativos, com nível de significância de $5 \%$.

\section{RESULTADOS}

Na intenção de identificar possíveis diferenças nas práticas de lazer entre rapazes e moças, optou-se por dividir a amostra em dois grupos. Considerando-se a escala de cinco pontos de Likert, optou-se por considerar hábitos de lazer consistentes no cotidiano dos adolescentes, somente aqueles que conseguissem alcançar valore iguais ou superior a 3 , designado desta forma que esta atividade é significativa e possui persistência temporal na vida dos mesmos.

Tabela 1. Características de hábitos de lazer entre os sexos

\begin{tabular}{|c|c|c|c|c|}
\hline Hábitos de lazer & $\hat{\gamma}=205$ & $\%$ & $Q=150$ & $\%$ \\
\hline 01 - Namorar ou conversar com amigos & 180 & 87,8 & 121 & 80,7 \\
\hline 02 - Ouvir música & 179 & 87,3 & 123 & 82,0 \\
\hline 03 - Assistir TV & 151 & 73,7 & 101 & 67,3 \\
\hline 04 - Navegar na Internet & 135 & 65,8 & 85 & 56,7 \\
\hline 05 - Leitura & 112 & 54,6 & 79 & 52,7 \\
\hline 06 - Praticar esportes sem orientação profissional & 77 & 37,6 & 52 & 34,7 \\
\hline 07 - Jogar vídeo game & 70 & 34,1 & 36 & 24,0 \\
\hline 08 - Visitar familiares ou pessoas conhecidas & 69 & 33,7 & 56 & 37,3 \\
\hline 09 - Praticar esportes com orientação profissional & 64 & 31,2 & 48 & 32,0 \\
\hline 10 - Cantar ou tocar algum instrumento & 54 & 26,3 & 37 & 24,7 \\
\hline 11 - Fazer trabalhos manuais & 48 & 23,4 & 34 & 22,7 \\
\hline 12 - Ir a bares e restaurantes. & 48 & 23,4 & 26 & 17,3 \\
\hline 13 - Apenas dormir ou descansar & 47 & 22,9 & 37 & 24,7 \\
\hline 14 - Assistir a eventos esportivos & 42 & 20,5 & 29 & 19,3 \\
\hline 15 - Participar de jogos de azar & 32 & 15,6 & 22 & 14,7 \\
\hline 16 - Ir ao cinema, shows, etc. & 31 & 15,1 & 22 & 14,7 \\
\hline 17 - Fazer compras & 21 & 10,2 & 20 & 13,3 \\
\hline 18 - Ir à igreja & 26 & 12,7 & 23 & 15,3 \\
\hline 19 - Fazer atividades de expressão corporal & 18 & 08,8 & 14 & 09,3 \\
\hline 20 - Ir ao shoping Center & 17 & 08,3 & 15 & 10,0 \\
\hline 21 - Realizar trabalhos de solidariedade social & 06 & 02,9 & 07 & 04,7 \\
\hline 22 - Participar de movimentos político-sociais. & 06 & 02,9 & 04 & 02,7 \\
\hline
\end{tabular}

$o^{\lambda}=$ Rapazes; + =Moças 
Apesar de se registrar pouca variação entre os principais hábitos de lazer adotados por rapazes e moças, verifica-se que os rapazes estão envolvidos com uma maior frequiência em hábitos de lazer tipicamente sedentários como, por exemplo, assistir TV, navegar na internet e jogar vídeo game e, que os mesmos apresentam uma maior participação na pratica de esportes sem orientação, por quanto às moças, com orientação profissional.

Estrategicamente, ao dividir a amostra entre aqueles que referiram praticar e não praticar atividades físico-desportivas nos momentos de lazer constatouse que os participantes do sexo feminino apresentaram uma maior prevalência de características de hábitos de lazer sedentário em relação ao sexo masculino, verificando-se $65,4 \%$ de hábitos ativos para os rapazes contra $59,3 \%$ de prevalência de hábitos inativos para as moças.

O passo seguinte adotado no estudo foi dividir a amostra de modo geral em dois grupos (lazer ativo e lazer sedentário) e verificar como se apresentava o comportamento dos mesmos em relação às práticas de lazer exaustivamente relatadas na literatura como influenciadoras de estilos de vida sedentários.

Figura 1. Distribuição de freqüência de hábitos de lazer sedentário entre os grupos

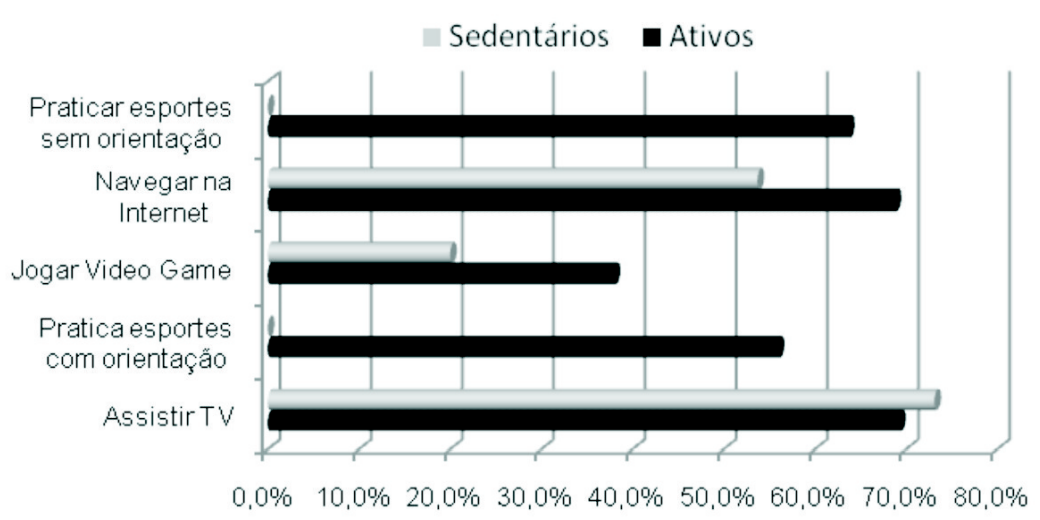

Como pode ser observado na Figura 1, os adolescentes de hábitos de lazer ativo apresentam uma maior freqüência nas práticas de lazer sedentário nas atividades de navegar na internet e jogar vídeo game. Considerando o tempo dedicado a essas atividades, buscou-se verificar o comportamento das variáveis entre adolescentes de hábitos de lazer ativo e sedentário. 
Figura 2. Tempo (minutos) dedicado aos hábitos de lazer sedentário entre os grupos

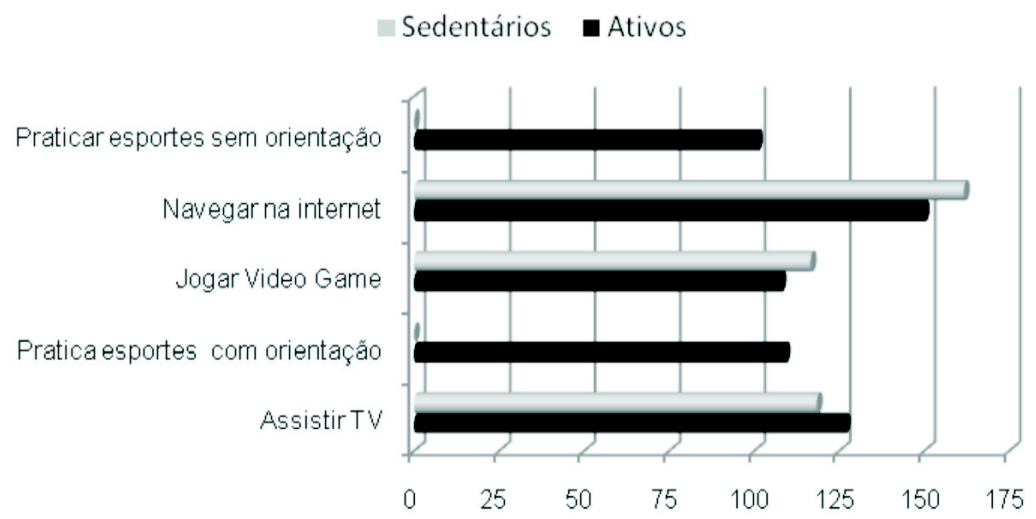

O estudo mostra um equilíbrio no tempo dedicado às práticas de lazer entre os adolescentes de hábitos ativo e sedentário, não havendo inclusive, diferenças significativas entre os grupos. A figura 2 ainda evidencia, que os adolescentes de hábitos de lazer ativo, encontram-se numa média de tempo superior a 100 minutos dedicadas a prática de atividades físico-despotivas no seu tempo livre.

O fato do indivíduo está envolvido com hábitos de lazer fisicamente pouco ativo, não garante que o mesmo possa ser caracterizado como sedentário, pois o mesmo pode estar envolvido em atividades cotidianas ou laborativas de médio a elevado esforço físico. Assim, ao analisar o nível de atividade física dos rapazes e das moças considerando seu envolvimento em práticas de lazer no tempo livre, constatou-se que $68,3 \%$ e $10,5 \%$ das moças referiram apresentar um nível de atividade física classificada como ativo e muito ativo respectivamente, contra $53,6 \%$ e $26,1 \%$ dos rapazes.

Como pode ser verificado na Tabela 2, o estudo mostra haver diferenças significativas quanto às características dos hábitos de lazer e nível de atividade física quando comparado entre os sexos a favor dos rapazes. 
Tabela 2. Comparação das características dos hábitos de lazer e do nível de atividade física entre os sexos

\begin{tabular}{ccc}
\hline Teste Estatístico & $\begin{array}{c}\text { Hábitos de } \\
\text { Lazer }\end{array}$ & $\begin{array}{c}\text { Nível de Atividade } \\
\text { Física }\end{array}$ \\
\hline Mann-Whitney U & 11998,500 & 11377,000 \\
Wilcoxon W & 33113,500 & 22108,000 \\
Z & $-4,000$ & $-4,210$ \\
\hline Nível de Significância &, 000 &, 000 \\
\hline
\end{tabular}

O fato de uma pessoa perceber-se como fisicamente ativa não garante que a mesma esteja envolvida em atividades nas quais respalde essa percepção, e a conduza a um nível de aptidão física aceitável (14). Visto essas observações, optou-se por verificar o comportamento das variáveis da aptidão física relacionada à saúde segundo o sexo e hábitos de lazer.

Tabela 3. Variáveis representativas da aptidão física relacionada à saúde

\begin{tabular}{|c|c|c|c|c|c|}
\hline Sexo & Variável & Hábitos de Lazer & $\mathbf{N}$ & Média & DP \\
\hline \multirow[t]{10}{*}{ Masculino } & $\operatorname{IMC}\left(\mathrm{Kg} / \mathrm{h}^{2}\right)$ & Ativo & 132 & 21,8 & 3,15 \\
\hline & & Sedentário & 73 & 21,4 & 5,52 \\
\hline & Gordura (\%) & Ativo & 132 & 13,5 & 4,10 \\
\hline & & Sedentário & 73 & 14,1 & 4,60 \\
\hline & Flexibilidade $(\mathrm{cm})$ & Ativo & 132 & 29,4 & 7,93 \\
\hline & & Sedentário & 73 & 28,5 & 8,25 \\
\hline & Força/Resistência ( $\mathrm{n} / \mathrm{min}$.) & Ativo & 128 & $42,4^{*}$ & 14,65 \\
\hline & & Sedentário & 73 & 34,3 & 8,69 \\
\hline & Resistência Aeróbica (9 min.) & Ativo & 116 & $1746,7^{*}$ & 285,76 \\
\hline & & Sedentário & 65 & 1590,1 & 319,50 \\
\hline \multirow[t]{10}{*}{ Feminino } & IMC $\left(\mathrm{Kg} / \mathrm{h}^{2}\right)$ & Ativa & 64 & 21,3 & 3,10 \\
\hline & & Sedentária & 85 & 21,5 & 3,03 \\
\hline & Gordura (\%) & Ativa & 63 & 21,1 & 3,28 \\
\hline & & Sedentária & 85 & 21,8 & 3,28 \\
\hline & Flexibilidade $(\mathrm{cm})$ & Ativa & 64 & $31,5^{*}$ & 6,68 \\
\hline & & Sedentária & 85 & 29,9 & 7,89 \\
\hline & Força/Resistência ( $\mathrm{n} / \mathrm{min}$.) & Ativa & 61 & 27,7 & 9,03 \\
\hline & & Sedentária & 81 & 24,6 & 7,02 \\
\hline & Resistência Aeróbica (9 min.) & Ativa & 57 & 1312,4 & 217,62 \\
\hline & & Sedentária & 71 & 1235,2 & 170,31 \\
\hline
\end{tabular}

Entre as variáveis representativas da aptidão física relacionada à saúde, somente no componente flexibilidade ocorreu um melhor desempenho por parte das moças. No entanto, o desempenho obtido por rapazes para esta variável foi suficiente para classificá-los como acima da zona saudável de aptidão física (PROESP-BR). 
Quanto à composição corporal, observaram-se valores idênticos de IMC para ambos os sexos, registrando-se inclusive classificação considerada dentro da zona saudável de aptidão física. No entanto, em virtude do critério de IMC vir recebendo severas críticas no meio científico (17), optou-se por identificar o percentual de gordura dos adolescentes, verificando-se assim que as moças apresentaram aproximadamente $8 \%$ de gordura corporal a mais que os rapazes. Um fato preocupante neste estudo, diz respeito às variáveis cujo desempenho está mais associado ao movimento ativo. Assim, por exemplo, o resultado obtido no teste que se propõe avaliar a força/ resistência, atribuiu classificação dentro da zona saudável apenas para os rapazes que praticam esportes nos momentos de lazer. Piores resultados foram observados no teste de resistência aeróbica, onde se constatou que nem mesmo os rapazes de hábitos de lazer ativo obtiveram classificação de níveis considerados dentro da zona saudável de aptidão física.

\section{DISCUSSÃO}

Os dados obtidos neste estudo revelam que praticamente não existem diferenças na adoção dos cinco principais hábitos de lazer referidos pelos jovens quando comparado em função do sexo, havendo apenas uma pequena troca na ordem de prioridade em que essas atividades são realizadas. O estudo revela, igualmente, que se trata de um dado importante por inclusive refletir uma característica de rapazes e moças de outras culturas $(11,12)$.

A maior prevalência dos rapazes em praticar atividades sem orientação profissional sugere que as moças apresentam uma maior dependência para realizar estas atividades, o que pode está associado à baixa percepção de competência para realizar atividades físico-desportivas sozinhas ou de não se sentirem confiantes para realizá-las em grupo (18). Outra observação importante neste estudo está relacionada à quebra de um paradigma proposto na literatura estabelecido nos últimos anos baseado numa relação de causa e efeito entre frequiência e tempo dedicado a assistir televisão, navegar na internet, jogar vídeo game e sedentarismo na infância e adolescência $(1,7,8,19,20)$. Recente estudo realizado nos Estados Unidos (21) respalda os achados deste trabalho. Desta forma, tudo leva a crer que o fenômeno lazer na adolescência deva ser mais bem investigado considerando os atributos pessoais dos adolescentes, bem como a influência dos contextos nos quais estes se encontram inseridos (22). 
O desempenho dos adolescentes nas variáveis orientadas à aptidão física relacionada à saúde quando comparado entre os sexos segue uma tendência evidenciada na literatura clássica $(10,23)$. A diferença no percentual de gordura observada neste estudo quanto ao sexo é considerada absolutamente normal visto as influencia das características maturacionais sobre a composição corporal (10).

Apesar de ter-se observado maiores diferenças na aptidão física relacionada à saúde entre os grupos a favor dos adolescentes do sexo masculino, o estudo sugere que sejam tomadas providências no sentido de desenvolver atividades que possam proporcionar a melhora da resistência geral dos adolescentes. Assim, o estudo permite concluir que mesmo os adolescentes de hábitos de lazer fisicamente ativo não necessariamente alcançarão resultados satisfatórios nas qualidades físicas que exijam movimento ativo, tais como resistência aeróbica e força/resistência abdominal e, que a implementação de programas de lazer e de condicionamento físico requerem atividades específicas. Dessa forma, independentemente do sexo, os adolescentes necessitam de ambas as atividades

\section{REFERÊNCIAS}

1. Alves JGB. Atividade física em crianças: promovendo a saúde do adulto. Rev. Bras. Saúde Mater. Infant. 2003; 3(1): 5-6.

2. BRASIL MS. Instituto Nacional do Câncer [Internet]. Inquérito domiciliar sobre comportamentos de risco e morbidade referida de doenças e agravos não transmissíveis. Disponível em www.inca.gov.br/inquerito. Consulta realizada em de maio de 2006.

3. Sportscotland [internet]. Increasing demand for sport and physical activity by girl. Disponível em www.sportscotland.org.uk. Consultado em maio de 2006.

4. Grieser M, Vu MB, Bedimo-Rung AL, Neumark-Stainer D, Moody J, Young D, Moe SG. Physical activity attitudes, preferences, and pratices in african american, hispanic, and caucasian girls. Healt Educ e Behav. 2006; 33(1): 40-51.

5. Lopes VP, Maia JAR. Actividade física nas crianças e jovens. Rev. Bras. Ciên. Mov. 2004; 6(1): 82-92.

6. Salles-Costa R, Heilborn ML, Werneck GL, Faerstein E, Lopes CS. Gênero e prática de atividade física de lazer. Cad. Saúde Pública. 2003; 19 (Sup.): 325-333.

7. Jenovesi JF, Bracco MM, Colugnati FAB, Taddei JAAC. Evolução no nível de atividade física de escolares observados pelo período de 1 ano. Rev. Bras. Cien. Mov. 2004; 12(1): $19-24$.

8. Matsudo VKR. "Construindo" saúde por meio da atividade física em escolares. Rev. Bras. Ciên. Mov. 2003; 11(4): 111-118.

9. Ford ES, Merritt RK, Heath GW, Powell KE, Washburn RA, Kriska A, et. al. Physical activity behaviors in lower and higher socioeconomic status populations. Am. J. Epidemiol. 1991; 133(12):1246 - 1256

10. Malina RM, Boucahrd C. Atividade física do atleta jovem: do crescimento à maturação. São Paulo: Roca. 2002. 
11. Cloes M, Ledent M, Didier P, Diniz J, Pieron M. Pratique et importance des principales activités de loisiris chez dês jeunes de 12 à 15 ans dans cinq pays européens. ADEP. 1997; 159/160: 51-60.

12. Esculcas C, Mota J. Actividade física e práticas de lazer em adolescentes. Rev. Port. Ciên. Desp. 2005; 5(1): 169-176.

13. Formiga NS, Ayroza I, Dias L. Escala das atividades de hábitos de lazer: construção e validação em jovens. Psic. 2005; 6(2): 71-79.

14. Guedes DP, Lopes CC, Guedes JERP. Reprodutibilidade e validade do questionário internacional de atividade física em adolescentes. Rev. Bras. Méd. Esporte. 2005; 11(2): 151-158.

15. Gaya A. [Internet]. PROESP-BR-Projeto Esporte Brasil: Indicadores de saúde e fatores de prestação esportiva em crianças e jovens. Disponível em: http://www.proesp.ufrgs.br/institucional/ PROESP-BR.pdf. Consulta feita em maio de 2005.

16. Petroski EL. Antropometria: técnicas e padronizações. $2^{\mathrm{a}}$ ed. Porto Alegre: Revista e Ampliada; 2003.

17. Ricardo DR, Araújo CGS. Índice de massa corporal: um questionamento científico. Arq. Bras. Cardiol. 2002; 79(1): 61-69.

18. Valentine NC, Toigo, AM. Ensinando Educação Física nas séries iniciais: desafios e estratégias. $2^{\underline{a}}$ ed. Canoas: Unilasalle; 2006.

19. Seabra AFT, Mendonça D, Garganta RM, Maia JAR. Influência de determinantes demográficobiológicos e sócio-culturais nos níveis de atividade física de crianças e jovens. Rev. Bras. Cine. Des. Hum. 2004; 6(2): 62-72.

20. Australian Bureaul of Statistics [Internet]. Children's participation in cultural and leisure activities. Disponível em http:/www.abs.gov.au/ausstats/abs@.nsf/mf/4901.0. Consulta feita em junho de 2006.

21. Taveras EM, Field AE, Berkey CS, Rifas-Shiman SL, Frazier AL, Colditz GA, et. al. Longitudinal rlationship between television viewing and leisure-time physical activity during adolescence. Pediatric. 2007; 119(2): 313-320.

22. Krebs RJ, Copetti F. As Propriedades da Pessoa na Perspectiva do Paradigma Bioecológico. Em: Silvia H Kr. (Org.). Ecologia do Desenvolvimento Humano-Pesquisa e Intervenção no Brasil. 1 ed. São Paulo: Casa do Psicólogo Livraria e Editora Ltda; 2004. pp. 67-89.

23. Gallahue DL, Ozmun JC. Compreendendo o desenvolvimento motor: bebês, crianças e adultos. $3^{\text {a }}$ ed. São Paulo: Phorte; 2005. 